\title{
Cost thresholds, cut-offs and sensitivities in stated choice analysis: identification and implications ${ }^{\star}$
}

\author{
Danny Campbell ${ }^{a} \quad$ David A. Hensher $^{b} \quad$ Riccardo Scarpa $^{c}$ \\ ${ }^{a}$ Gibson Institute for Land, Food and Environment, School of Biological Sciences, and \\ Institute for a Sustainable World, Queen's University Belfast \\ ${ }^{b}$ Institute of Transport and Logistics Studies, The Business School, University of Sydney \\ ${ }^{c}$ Waikato Management School, The University of Waikato, Hamilton, New Zealand and \\ CenSoC, University of Technology of Sydney, Australia
}

February 2011

Prepared for the $2^{\text {nd }}$ International Choice Modelling Conference

\section{Abstract}

Within the discrete choice literature, there is growing recognition that some respondents do not process all attributes when evaluating their choice outcomes. Worryingly, the cost attribute is often among those attributes that are likely to be ignored by respondents. We use probabilistic decision process models (similar in form to latent class models, but where we define the classes to describe specific heuristics) to facilitate situations where respondents adopt cost thresholds and cutoffs. We further develop this model to address the potential confounding between preference heterogeneity and processing heterogeneity by simultaneously allowing for a segmentation of respondents based on their sensitivities to cost. Results, based on an empirical dataset on the existence value of rare fish species in Ireland, provide further confirmation that a share of respondents did not attended to cost. Importantly, however, when heterogeneity to cost levels is accounted for the inferred incidence of complete non-attendance is markedly lower, to the extent that when cost thresholds and cut-offs are also accommodated it almost disappears. This modelling approach leads to significant gains in model fit and has important implications for welfare analysis.

Keywords: stated choice analysis, cost thresholds, cost cut-offs, cost sensitivities, latent class model, probabilistic decision process model, existence value

*Acknowledgements: The authors wish to acknowledge the funding provided by the EU INTERREG IIIA Programme for Ireland/Northern Ireland for data collection. 


\section{Introduction}

Within the context of stated choice experiments, when offered a choice between a bundle of goods, respondents are expected to choose the good that delivers them the highest utility. Central to this, is the assumption that respondents evaluate the relative merits of attributes across all alternatives. However, there is growing recognition that some respondents do not process all attributes when evaluating their choice outcomes (e.g., see Hensher et al., 2005; Campbell et al., 2008; Hensher, 2010; Scarpa et al., 2009; Carlsson et al., 2010; Scarpa et al., 2010). Worryingly, the cost attribute is often among those attributes that have been ignored by respondents. This can have serious implications since the parameter estimate for cost is used to derive estimates of welfare change (such as marginal willingness to pay (MWTP)), which are often the main goal of stated choice studies, especially in nonmarket valuation.

A range of factors may give rise to such attribute non-attendance, including choice task complexity (e.g., DeShazo and Fermo, 2002; Puckett and Hensher, 2008), unfamiliarity with the good at hand (e.g., Campbell et al., 2008) and the fact that the attributes are not behaviourally relevant to certain respondents (e.g., Sælensminde, 2006). In addition, as discussed in Carlsson et al. (2010), the design itself can induce lexicographic orderings, particularly in situations where the cost attribute is not high enough to result in a tradeoff for the respondent. As suggested by Puckett and Hensher (2008), the range and relative equivalence of the cost attribute levels among alternatives in a particular choice task may also lead respondents to ignore the cost attribute in some choice tasks and not in others.

To date, studies using econometric models addressing non-attendance have primarily focussed on identifying non-attendance at the attribute level and ignored the possibility that attendance might be dependent on the levels of the attribute in question. In other words the common assumption has been that if an attribute is not attended to by a respondent then this takes place at every level of the attribute in question. However, at least in principle, one may postulate the hypothesis that due to differences in budget constraints - and hence to differences in the marginal utility of money - there is likely to be heterogeneity in the way respondents process the cost information (e.g., see Campbell, Aravena and Hutchinson, 2011). Respondents may have cost thresholds and cut-offs (e.g., Swait, 2001; Han et al., 2001; Cantillo et al., 2006; Cantillo and Ortúzar, 2006; Chou et al., 2008; Mørkbak et al., 2010) and exclude the cost information of those alternatives that are outside their threshold (e.g., Mørkbak et al., 2010). The premise behind our study is that respondents will obviously treat attribute levels that fall outside of their cost thresholds differently from those within them, and in many cases it is likely that levels that are not within their consideration range will play no part on their choice. Consequently, estimates of non-attendance associated with the cost parameter that do not identify such behaviour tend to overestimate non-attendance 
to cost. Rather than including all cost levels in their decision-making process, there may be a subset of respondents who attend to the cost levels that are within their consideration set (i.e. within the thresholds of relevance), as well as the conventional subset whose choices are not influenced by any of the cost levels.

To accommodate this range of processing strategies, in this paper we develop a probabilistic decision process model. This is similar in form to a latent class model, but the classes here are meant to describe a specific heuristic. The latent class model is hence a tool to implement the complete range of cost thresholds that may be held by respondents. However, we are cognisant of the need to distinguish non-attendance from situations where respondent's dis-utility for cost is relatively small (i.e., where they are relatively price (cost) insensitive, at least for the good at hand). Both behaviours will produce cost coefficients that are close to zero, but their interpretation will differ, especially from the welfare analysis viewpoint. To address this potential confounding we simultaneously allow for a segmentation of respondents based on sensitivities to cost and their adoption of non-attendance processing and thresholds dependent on cost levels.

This paper intends to widen the discussion on non-attendance, and is motivated by the fact that greater behavioural insights into the levels of cost that actually have an influence on respondent's choices should help both at the experimental design stage (e.g., see Hensher et al., 2011, for a discussion) and at the analytical stage, particular when it comes to welfare analysis. Results, based on an empirical dataset exploring the existence value of rare fish species in Ireland, provide further confirmation that a share of respondents excluded information concerning the level of cost. Importantly, however, the incidence of complete non-attendance is found to be markedly lower, to the extent that it almost disappears, when the range in cost thresholds and cut-offs are accounted for at the same time as the range in the dis-utilities towards the cost attribute (i.e., sensitivities to cost) among the sample are facilitated. Moreover, we find compelling evidence for the need to disentangle heterogeneity in cost sensitivities and heterogeneity in the adoption of cost thresholds. Results further show that the gains in model fit to be achieved are substantive and that the implications for willingness to pay when both types of heterogeneity are addressed are of relevance to practitioners.

The remainder of the paper is structured as follows: Section 2 describes our modelling approach; Section 3 outlines our empirical case-study; Section 4 presents the results from the analysis; and, Section 5 concludes.

\section{Modelling approach}

Starting with the conventional specification of utility, where respondents are indexed by $n$, preferred alternatives in the choice task by $i$, choice occasions by $t$ and the cost and non-cost attributes are represented by $p$ and $x$ respectively, we 
have:

$$
U_{n i t}=\alpha p_{n i}+\sum_{k=1}^{K} \beta_{k} x_{k n i t}+\varepsilon_{n i t},
$$

where $\alpha$ and $\beta$ are parameters to be estimated for the cost and $K$ non-cost attributes respectively, $\varepsilon$ is an iid type I extreme value (EV1) distributed error term, with constant variance $\pi^{2} / 6$, and where we have assumed a conventional linearin-parameters specification of the observed utility function. Given these assumptions, the probability of the sequence of choices made by individual $n$ can be represented by the following multinomial logit (MNL) model:

$$
\operatorname{Pr}\left(y_{n} \mid p_{n}, x_{n}\right)=\prod_{t=1}^{T_{n}} \frac{\exp \left(\alpha p_{n i}+\sum_{k=1}^{K} \beta_{k} x_{k n i t}\right)}{\sum_{j=1}^{J} \exp \left(\alpha p_{n j}+\sum_{k=1}^{K} \beta_{k} x_{k n j t}\right)},
$$

where $y_{n}$ gives the sequence of choices over the $T_{n}$ choice occasions for respondent $n$, i.e., $y_{n}=\left\langle i_{n 1}, i_{n 2}, \ldots, i_{n T_{n}}\right\rangle$.

In this paper we are interested in explaining the heterogeneous nature of sensitivities to cost among the sample of respondents. Such (unobserved) heterogeneity of marginal utility of money is typically accommodated assuming a random distribution for the cost coefficient. Rather than a continuous random distribution, we opt for a finite one. The advantage of this non-parametric approach is that commonly used continuous distributions are often unsuitable for representing the distribution of cost sensitivities. Finite distributions-instead-can provide greater flexibility and have practical appeal as the results can have more intuitive meaning than the parameter and moments of the distributions retrieved from continuous parametric distributions. Since our focus in this paper is exploring probabilistic decision processes (PDPs) in the form of patterns of cost sensitivities, we use a latent class (LC) model representation, in which class-specific parameters are obtained only for the cost coefficient:

$$
\operatorname{Pr}\left(y_{n} \mid p_{n}, x_{n}\right)=\sum_{c=1}^{C} \pi_{c} \prod_{t=1}^{T_{n}} \frac{\exp \left(\alpha_{c} p_{n i}+\sum_{k=1}^{K} \beta_{k} x_{k n i t}\right)}{\sum_{j=1}^{J} \exp \left(\alpha p_{n j}+\sum_{k=1}^{K} \beta_{k} x_{k n j t}\right)},
$$

where $\alpha_{c}$ represents a class-specific coefficient for the cost attribute, and $\pi_{c}$ denotes the probability associated with class $c$.

While the specification in Equation 3 helps uncover the distribution of sensitivities to cost within the sample, it neglects the fact that there is likely to be confounding between the heterogeneity in cost sensitivities and heterogeneity in 
the attention respondents allocated to the cost attribute. Indeed, a relatively small (in absolute terms) coefficient of marginal utility will be retrieved for respondents who paid little attention to the cost attribute as well as for those with low marginal utility of money. Failing to recognise this, however, will lead to a misleading interpretation of the distribution of cost sensitivities within the sample of respondents. Importantly, since the coefficient for the cost attribute appears in the denominator of the MWTP calculation, it is important to distinguish between the two types of heterogeneity.

A further attractive feature of finite representations is their ability to identify and accommodate heterogeneity in the processing strategies adopted by respondents (e.g., see Scarpa et al., 2009; Campbell, Aravena and Hutchinson, 2011; Hensher and Greene, 2010; Campbell, Hensher and Scarpa, 2011, for applications). In this paper we exploit this feature to derive probabilistic estimates of the heuristics adopted by respondents. In practice, we retrieve these estimates by appropriately defining the values that the parameters can take within each of the latent classes. While based on a LC model specification, we prefer to describe such models as PDP models, since each latent class is described by a specific heuristic rather than marginal utilities. In this paper, we estimate a PDP model where the value of $\alpha$ in one of the classes is fixed to zero. Doing so, ensures that the processing heterogeneity is accommodated and the value of $\pi$ associated with this class gives an indication of the proportion of the sample where cost had no bearing on the observed sequence of choices. In an attempt to concurrently recognize the fact that there may also be heterogeneous sensitivities to cost among respondents who do not ignore the cost attribute, we obtain additional mass points for the cost coefficient using specifications where more than one class is assumed as having a non-zero cost coefficient.

Despite the appeal of this approach for accommodating respondents who ignored the cost attribute, it may also be the case that respondents ignored only a subset of the cost levels. Furthermore, as documented and investigated in Campbell, Aravena and Hutchinson (2011); Mørkbak et al. (2010), there is likely to be heterogeneity in the subsets of cost levels that actually influenced respondent's choices. For instance, some respondents may have had cost thresholds, whereby cost only influenced their choices if it fell within a certain threshold (e.g., see Hensher and Rose, 2011). Similarly, some respondents may have employed cut-offs, whereby they disregarded prices that fell below their lower limit or went beyond their upper limit. To deal with such processing strategies we introduce a further PDP model specification:

$$
\operatorname{Pr}\left(y_{n} \mid p_{n}, x_{n}\right)=\sum_{c=1}^{C} \pi_{c} \prod_{t=1}^{T_{n}} \frac{\exp \left(\sum_{l=1}^{L} \alpha_{l c} p_{n i}\left(\delta_{1}+\delta_{2}+\ldots+\delta_{L}\right)+\sum_{k=1}^{K} \beta_{k} x_{k n i t}\right)}{\sum_{j=1}^{J} \exp \left(\sum_{l=1}^{L} \alpha_{l c} p_{n j}\left(\delta_{1}+\delta_{2}+\ldots+\delta_{L}\right)+\sum_{k=1}^{K} \beta_{k} x_{k n j t}\right)}
$$


where $\delta_{l}$ is a dummy variable, which equals to one if the cost of the alternative is denoted with level $l$ and zero otherwise, and $\alpha_{l c}$ is the parameter associated with this level in class $c$. This differs from Equation 2 and Equation 3 where $\alpha$ takes the same values for all $L$ levels. However, the advantage of specifying separate parameters for the various cost levels is that we can construct a PDP model to accommodate the fact that respondents may apply thresholds and cuts-off and, hence, consider only a subset of levels of the cost attribute.

For instance, consider the scenario where the cost attribute is described with three levels (i.e., $L=3$ ), as follows: $p_{\text {Low }} ; p_{\text {Middle }}$; and, $p_{\text {High }}$. Under such a scenario, aside from where respondent ignores cost independently of its levels, six patterns of cost thresholds and cut-offs can be identified:

1. respondent considers only the Low cost level (i.e., threshold is equal to the Low level);

2. respondent considers the Low and Middle cost levels (i.e., threshold is between Low and Middle, where the Middle level represents the upper cutoff);

3. respondent considers all cost levels (i.e., the complete range of the cost levels are within the threshold);

4. respondent considers only the Middle cost level (i.e., threshold is equal to Middle level);

5. respondent considers the Middle and High cost levels (i.e., threshold is between Middle and High and the lower cut-off is equal to the Middle level); and,

6. respondent considers only the High cost level (i.e., threshold is equal to the High level). ${ }^{1}$

These six patterns can be dealt with using a PDP model with seven classes. The first class sets $\alpha_{\text {Low }}, \alpha_{\text {Middle }}$ and $\alpha_{\text {High }}$ to zero to accommodate situations where cost is non-attended to, regardless of its levels. Classes 2-7 are specified to retrieve probabilistic estimates of the six patterns of cost thresholds and cut-offs as follows: $\alpha_{\text {Low }}$ is restricted to zero in classes 5-7; $\alpha_{\text {Middle }}$ is specified as zero in classes 2 and 7; and, $\alpha_{\text {High }}$ is fixed to zero in classes 2, 3 and 5. In estimation, allowing $\alpha$ to take non-zero values and constraining $\alpha_{\text {Low }}=\alpha_{\text {Middle }}=\alpha_{\text {High }}$ across classes 2-7 ensures that cost has a linear and continuous effect on utility, which is a typical assumption used in discrete choice modelling, but it allows the levels of cost that actually have influenced the respondent's sequence of choices to be estimated by maximization of the log-likelihood function of the sample. In this manner prob-

\footnotetext{
${ }^{1}$ A further scenario is possible whereby respondents consider the Low and High levels only. For the purposes of this paper we consider only scenarios in which the threshold does not consist of any breaks, which is likely to be a more realistic event. In this case, the number of cost threshold and cut-off patterns can be identified using $\sum_{l=1}^{L} l$.
} 
abilistic estimates of the heuristics adopted by respondents are obtained directly from the data.

While this accounts for the thresholds and cut-offs, it assumes that those respondents who did consider the cost level(s) had homogeneous sensitivities to cost. This is unlikely to be the case due to the prevalence of differences in marginal utility of money across respondents. We, therefore, add further classes to the model. Using the example given above, we add an additional six classes similar in nature to those specified in classes 2-7, but where the non-zero value of $\alpha$ for classes $8-14$ is different from that obtained for classes $2-7$. This yields probabilistic estimates of the heuristics adopted by respondents with a different sensitivity to cost.

\section{Empirical case-study}

To illustrate the proposed methodology on an empirical case-study we use stated preference data collected to estimate the existence value of rare and endangered fish species in the Lough Melvin Catchment in Ireland. Lough Melvin is a freshwater lake in the North West of Ireland which straddles the border between Northern Ireland and the Republic of Ireland. With a unique population of native fish species, the Lough Melvin Catchment has an internationally important conservation status. Lough Melvin and its associated river system supports the only remaining population of Arctic char Salvelinus alpinus (L.) in Northern Ireland and contains Atlantic salmon Salmo salar (L.) and three genetically distinct populations of brown trout known as ferox Salmo ferox (L.), gillaroo Salmo stomachicus (L.) and sonaghan Salmo nigripinnis (L.). Since the habitat of these fish populations is recognised as being vulnerable, there is a need to assess the extent to which the general public supports the prevention of their extinction.

The stated choice experiment consisted of a panel of sixteen repeated choice tasks. Each choice task outlined three possible outcomes. The first two outcomeslabelled as 'Option A' and 'Option B'-described the conservation status of each of the fish species after the implementation of two experimentally designed conservation schemes. At the end of these conservation efforts, the fish species would either be 'Conserved' or 'Extinct'. While a particular scheme described under either 'Option A' or 'Option B' may have been unable to prevent some of the fish species from becoming extinct, they both ensured against the extinction of all fish species (i.e., at least one species was conserved under each scheme). The final outcome-labelled as 'Do Nothing' - showed the expected outcome if nothing was done to protect the fish species. In this case, the respondents were informed that all five fish species would become extinct. 'Option A' and 'Option B' were both described to respondents as available at a positive cost, using one of the following cost levels: $€ 3$, €6, €12, €24 or $€ 48$. The payment vehicle used was 
the amount that they would personally have to pay per year - through an increase in their Income Tax and/or Value Added Tax contributions-to implement the scheme. The 'Do Nothing' (or status quo) option had zero cost to the respondent. The experimental design was obtained using an algorithm that minimized the variance of the sum of the MWTPs (Scarpa and Rose, 2008) and invoked Bayesian assumptions informed on estimates from pilot studies (Vermeulen et al., 2011).

The population of interest was the adult population of the Republic of Ireland and Northern Ireland. The study adopted a stratified random sample to reflect the geographic distribution of the adult population; the approximate rural/urban split; the approximate socio-economic status of the regional population; and the approximate gender and age profile of the populations within both jurisdictions. A final sample of 624 usable interviews was obtained which, with each respondent answering 16 choice tasks, resulted in 9,984 observations for model estimation. For further analysis on this data interested readers are referred to Campbell et al. (2010); Campbell and Hess (2011).

\section{Results}

\subsection{Estimation of a PDP with cost-specific latent classes}

As a point of reference our analysis starts with the MNL model (Equation 2), with marginal utility parameters for the $\operatorname{cost}(\alpha)$ and fish attributes $\left(\beta_{\mathrm{AC}}\right.$ for Arctic char; $\beta_{\mathrm{AS}}$ for Atlantic salmon; $\beta_{\mathrm{F}}$ for ferox; $\beta_{\mathrm{G}}$ for gillaroo; and, $\beta_{\mathrm{S}}$ for sonaghan) and an alternative specific constant for the status quo option $\left(\beta_{\mathrm{SQ}}\right)$, whose coefficient can be interpreted as the marginal (dis-)utility for the extinction of all fish species (whereas, the parameters associated with each fish species represents the marginal utility associated with preserving the species). Inspecting the results obtained from this model in Table 1, we note, as anticipated, that the fish coefficients are estimated with positive, and statistically significant, sign-implying that respondents, all else held constant, prefer that each of the fish species are conserved rather than go extinct. In line with a-priori expectations, the cost and status quo coefficients are both estimated as having negative, and significant, signsimplying that respondents, ceteris paribus, prefer policy scenarios that are (i) less expensive and (ii) prevent the extinction of all the fish species, respectively.

Table 1 also reports results from two latent class specifications (Equation 3) in which the distribution of cost sensitivities is firstly assumed as having two mass points (LC-2) and subsequently with three mass points (LC-3). Under both models similar inferences as those reached under the MNL can be made regarding the coefficients for the fish attributes and status quo constant. Of greater interest are the retrieved distributions of cost sensitivities. Results obtained from LC-2 suggest that over 70 percent of respondents have a marginal utility for cost that is not 
Table 1: Estimation results (MNL and LC models)

\begin{tabular}{|c|c|c|c|c|c|c|}
\hline & \multicolumn{2}{|c|}{ MNL } & \multicolumn{2}{|c|}{ LC-2 } & \multicolumn{2}{|c|}{ LC-3 } \\
\hline & est. & $\mid t$-rat. $\mid$ & est. & $\mid t$-rat. $\mid$ & est. & $\mid t$-rat. $\mid$ \\
\hline$\alpha_{c_{1}}$ & -0.018 & 20.51 & 0.002 & 1.37 & 0.003 & 3.00 \\
\hline$\alpha_{c_{2}}$ & - & - & -0.118 & 26.75 & -0.089 & 27.76 \\
\hline$\alpha_{c_{3}}$ & - & - & - & - & -0.361 & 21.28 \\
\hline$\beta_{\mathrm{AC}}$ & $\overline{0.30} \overline{4}$ & 13.24 & 0.294 & $\overline{1} 1.83$ & $\overline{0} . \overline{3} 0 \overline{7}^{-}$ & $\overline{12} . \overline{13}$ \\
\hline$\beta_{\mathrm{AS}}$ & 0.642 & 24.82 & 0.666 & 23.83 & 0.681 & 24.00 \\
\hline$\beta_{\mathrm{F}}$ & 0.297 & 12.83 & 0.297 & 11.91 & 0.304 & 12.03 \\
\hline$\beta_{\mathrm{G}}$ & 0.355 & 15.40 & 0.395 & 15.78 & 0.408 & 16.17 \\
\hline$\beta_{\mathrm{S}}$ & 0.508 & 20.56 & 0.548 & 20.35 & 0.566 & 20.75 \\
\hline$\beta_{\mathrm{SQ}}$ & -0.974 & 20.59 & -1.556 & 26.76 & -1.873 & 29.32 \\
\hline$\pi_{\alpha_{c_{1}}}$ & $\overline{1.000}$ & fixed & $0 . \overline{71} \overline{3}$ & $3 \overline{6} . \overline{7} 6$ & $0 . \overline{6} \overline{7} 0^{-}$ & $\overline{3} 3 . \overline{5} 0$ \\
\hline$\pi_{\alpha_{c_{2}}}$ & - & - & 0.287 & 14.79 & 0.247 & 13.18 \\
\hline$\pi_{\alpha_{c_{3}}}$ & - & - & - & - & 0.083 & 7.01 \\
\hline $\mathcal{L}(\hat{\beta})$ & \multicolumn{2}{|c|}{$-8,727.64$} & \multicolumn{2}{|c|}{$-7,361.20$} & \multicolumn{2}{|c|}{$-7,115.40$} \\
\hline$K$ & \multicolumn{2}{|c|}{7} & \multicolumn{2}{|c|}{9} & \multicolumn{2}{|c|}{11} \\
\hline $\bar{\rho}^{2}$ & \multicolumn{2}{|c|}{0.204} & \multicolumn{2}{|c|}{0.328} & \multicolumn{2}{|c|}{0.350} \\
\hline $\mathrm{AIC} / \mathrm{N}$ & \multicolumn{2}{|c|}{1.750} & \multicolumn{2}{|c|}{1.476} & \multicolumn{2}{|c|}{1.428} \\
\hline $\mathrm{BIC} / \mathrm{N}$ & \multicolumn{2}{|c|}{1.755} & \multicolumn{2}{|c|}{1.483} & \multicolumn{2}{|c|}{1.436} \\
\hline
\end{tabular}

significantly different from zero, and that the remaining respondents have a relatively high marginal utility for money (disutility for cost). Similarly, under LC-3 the marginal utility of cost is close to zero for almost 70 percent. Worryingly, we also observe that the coefficient for this subset of respondents is estimated to be positive and significant, but only very slightly higher than zero (0.03). From LC-3 we also find that approximately 8 percent of respondents have a relatively high marginal utility for money, and that almost one-quarter of them have a moderate one. We observe that allowing for heterogeneous sensitivities to cost leads to an improvement in model fit compared to the MNL model. As one moves from LC-2 to LC-3 we also note an improvement of almost 250 log-likelihood units at the expense of fitting two additional parameters. The null embedded in LC-2 provides a likelihood ratio test statistic of 491.61 against the $\chi^{2}$ critical value of $5.99\left(\chi_{2,0.05}^{2}\right)$. Altogether this first set of latent class models suggests high heterogeneity of preference for the cost attribute, in keeping with our expectation. We now turn to a more in-depth and better structured investigation of this issue by first introducing cost non-attendance via adequate PDPs in the latent class structure of the model. 


\subsection{Cost non-attendance and cost heterogeneity}

A standard interpretation of the results attained under the LC-2 and LC-3 specifications would signal that a large share of the sample had zero (or close to zero) marginal utilities for the cost attribute. While this may be the case, it is more likely that the cost attribute was ignored by these respondents, and thus, any interpretation of their marginal utilities for cost may be inappropriate. We note that it can be difficult to make this distinction. Nevertheless, it makes sense to constrain the cost coefficient to zero in one class as this accommodates cases where respondents have zero marginal utility for cost and/or cases where they have not attended to the attribute (which in both cases means that the cost of alternatives does not have any bearing on the sequence of choices made by respondents). For this reason we estimate three PDP models which aim at accommodating the nonattendance of the cost attribute via fixing the cost coefficient to zero in the first class. In an attempt to also uncover some of the heterogeneity in cost sensitivities among respondents who did attend to the cost attribute, we compare specifications with one, two and three non-zero mass points for cost (labelled PDP-1, PDP-2 and PDP-3 respectively). Results of these are presented in Table 2.

Again, inferences relating to the estimated coefficients for the fish and status quo constant are in line with those in the previous models. Starting with PDP1 , we note that it is very close, in terms of model fit and estimated parameters, to LC-2. This is not surprising, given the fact that the cost coefficient was not significantly different from zero in the first class in LC-2. Moving to PDP-2, we again find that it produces quite comparable results to those attained in LC-3, which can be explained by the lowly estimated cost coefficient in the first class in LC-3. Given the relative equivalence of these models to their standard LC model counterparts, we remark that one degree of freedom has been saved (which is alluded to by the slight lowering in the BIC statistic). More importantly, though, we highlight that the interpretation of the two sets of models are different, and that the implied welfare estimates will be entirely different under the two approaches. Under PDP-3 we find that for almost two-thirds of respondents, the cost attribute had no influence on their choices. Looking at the heterogeneity in cost sensitivities among the remaining respondents reveals that a relatively small subset (approximately 5 percent of the sample) had very high marginal utility for money. The other predicted mass points are less extreme and have quite comparable class membership probabilities (just over 15 percent for each). Compared to PDP-1 and PDP-2, the model fit achieved under PDP-3 is much superior. Respectively, at the expense of four and two additional parameters there is a reduction of 312 and 70 log-likelihood units, which contributes to significant likelihood ratio test statistics.

We remark that accounting for more of the heterogeneity contributes to a decline in the estimated incidence of respondents identified as having ignored the cost attribute. This is because models explaining more of the preference hetero- 
Table 2: Estimation results (PDP models)

\begin{tabular}{|c|c|c|c|c|c|c|}
\hline & \multicolumn{2}{|c|}{ PDP-1 } & \multicolumn{2}{|c|}{ PDP-2 } & \multicolumn{2}{|c|}{ PDP-3 } \\
\hline & est. & $\mid t$-rat. $\mid$ & est. & $\mid t$-rat. $\mid$ & est. & $\mid t$-rat. $\mid$ \\
\hline$\alpha_{c_{1}}$ & 0.000 & fixed & 0.000 & fixed & 0.000 & fixed \\
\hline$\alpha_{c_{2}}$ & -0.121 & 28.47 & -0.093 & 30.11 & -0.153 & 17.74 \\
\hline$\alpha_{c_{3}}$ & - & - & -0.368 & 21.76 & -0.063 & 14.92 \\
\hline$\alpha_{c_{4}}$ & - & - & - & - & -0.492 & 14.87 \\
\hline$\beta_{\mathrm{AC}}$ & $0.2 \overline{9} \overline{9}$ & 12.13 & $0 . \overline{317}$ & $12 . \overline{6} 4$ & $0 . \overline{3} 36$ & $\overline{13} . \overline{19}$ \\
\hline$\beta_{\mathrm{AS}}$ & 0.678 & 25.49 & 0.709 & 26.37 & 0.737 & 26.52 \\
\hline$\beta_{\mathrm{F}}$ & 0.303 & 12.40 & 0.318 & 12.81 & 0.334 & 13.25 \\
\hline$\beta_{\mathrm{G}}$ & 0.398 & 15.99 & 0.418 & 16.72 & 0.431 & 16.98 \\
\hline$\beta_{\mathrm{S}}$ & 0.555 & 21.11 & 0.585 & 22.01 & 0.610 & 22.21 \\
\hline$\beta_{\mathrm{SQ}}$ & -1.561 & 26.85 & -1.878 & 29.35 & -1.952 & 29.67 \\
\hline$\pi_{\alpha_{c_{1}}}$ & $\overline{0} \overline{7} \overline{1} \overline{8}$ & $37.5 \overline{8}$ & $0 . \overline{6} 7 \overline{8}$ & $\overline{3} \overline{4} . \overline{4} 4$ & $\overline{0} . \overline{6} \overline{4} 3^{-}$ & $\overline{2} \overline{9} . \overline{4} \overline{8}$ \\
\hline$\pi_{\alpha_{c_{2}}}$ & 0.282 & 14.78 & 0.239 & 13.08 & 0.154 & 8.51 \\
\hline$\pi_{\alpha_{c_{3}}}$ & - & - & 0.082 & 6.97 & 0.152 & 8.05 \\
\hline$\pi_{\alpha_{c_{4}}}$ & - & - & - & - & 0.051 & 5.06 \\
\hline $\mathcal{L}(\hat{\beta})$ & \multicolumn{2}{|c|}{$-7,362.11$} & \multicolumn{2}{|c|}{$-7,119.88$} & \multicolumn{2}{|c|}{$-7,050.22$} \\
\hline$K$ & \multicolumn{2}{|c|}{8} & \multicolumn{2}{|c|}{10} & \multicolumn{2}{|c|}{12} \\
\hline $\bar{\rho}^{2}$ & \multicolumn{2}{|c|}{0.328} & \multicolumn{2}{|c|}{0.350} & \multicolumn{2}{|c|}{0.356} \\
\hline $\mathrm{AIC} / \mathrm{N}$ & \multicolumn{2}{|c|}{1.476} & \multicolumn{2}{|c|}{1.428} & \multicolumn{2}{|c|}{1.415} \\
\hline $\mathrm{BIC} / \mathrm{N}$ & \multicolumn{2}{|c|}{1.482} & \multicolumn{2}{|c|}{1.435} & \multicolumn{2}{|c|}{1.423} \\
\hline
\end{tabular}

geneity are better equipped to disentangle respondents who are relatively costinsensitive from those who did not attend to price. This provides a strong indication of the confounding between preference heterogeneity and non-attendance, and the need to accommodate both concurrently in order to draw appropriate inferences and reliable welfare estimates.

\subsection{An extension: heterogeneity in cost levels}

Notwithstanding the behavioural insights afforded by the PDP models reported in Table 2, they are based on the assumption that respondents uniformly ignored or attended to cost regardless of its levels. But it is conceivable that some respondents considered a subset of the cost levels (e.g., by employing cost thresholds and cutoffs rules) and that, furthermore, there is heterogeneity in the subsets of cost levels that actually influenced respondent's choices. To deal with this we stay within the PDP modelling framework, but estimate membership probabilities for the various patterns of cost thresholds and cut-offs (Equation 4). 
Given the five non-zero cost levels used in our empirical study (i.e., $€ 3$, $€ 6$, $€ 12$, €24 or $€ 48$ ), and conditional on the respondent not completely neglecting the cost attribute, there are 15 (i.e., $\sum_{l=1}^{5} l=1+2+3+4+5$ ) distinct patterns of thresholds and cut-offs that do not involve any gaps between levels that could have been used by respondents. The PDP (threshold) (PDP(T)) models reported in Table 3 are structured to facilitate the estimation of the probabilities associated with each of these patterns. This is achieved using different cost coefficients for each cost level in order to correspond with the different threshold patterns. Cost coefficients are either constrained to be zero or unconstrained in estimation. When allowed to be different from zero though, they are all constrained to take the same value across cost levels so as to ensure the same linear effect. To also account for possible heterogeneity in cost sensitivities among respondents who did attend to price, we also compare specifications with one, two and three non-zero mass points for cost (labeled PDP(T)-1, PDP(T)-2 and PDP(T)-3, respectively).

An examination of the point estimates obtained for the fish attributes and status quo constant provides similar inference across the three PDP(T) model specifications, which are also in line with those previously discussed. Turning our attention to PDP(T)-1, the predicted class membership probability of the first class in which the cost coefficient for all cost levels is restricted to zero, suggests that just less than 60 percent of respondents did not attend to any cost level. We note that this is a smaller proportion than estimated in any of the previous models, insinuating that the phenomenon of neglecting the cost attribute may not be as high as implied by previous model estimates. While the estimates obtained from PDP(T)- 1 suggest that a non-zero cost coefficient is attainable for over 40 percent of respondents, the model predicts that only approximately 12 percent of respondents considered all cost levels uniformly when making their choices. On this basis, almost 30 percent of respondents are inferred to exploit cost thresholds and/or cut-offs. Interestingly, 13 percent (i.e., $0.073+0.001+0.001+0.002+0.053$ ) of respondents focused on only one cost level, the majority of whom only attended to the lowest cost level. Adding together the class membership probabilities in which the coefficient for the $€ 3$ cost level is non-zero, reveals that almost 24 percent (i.e., $0.073+0.037+0.005+0.001+0.124)$ considered this cost level. With the corresponding figures for the $€ 6, € 12$, $€ 24$ or $€ 48$ cost levels at 19 percent, 22 percent, 24 percent and 28 percent respectively, we remark that there appears to be a general rise in attendance to cost as its level increases, as one would expect. We also report that 11 percent of respondents only considered the two lowest cost levels, and that this percent modestly increases (by less than 2 percent) when it is extended to also include all but the highest cost. The proportion of respondents who considered only the two highest cost levels is estimated at 8 percent, whereas 15 percent and 17 percent of respondents considered only the highest three and four cost levels, respectively. Comparing the model fit obtained under PDP(T)-1 to PDP-1, we remark a improvement of 46 log-likelihood units at the expense of 
Table 3: Estimation results (PDP(T) models)

\begin{tabular}{|c|c|c|c|c|c|c|}
\hline & \multicolumn{2}{|c|}{ PDP(T)-1 } & \multicolumn{2}{|c|}{ PDP(T)-2 } & \multicolumn{2}{|c|}{ PDP(T)-3 } \\
\hline & est. & $\mid t$-rat. $\mid$ & est. & $\mid t$-rat. $\mid$ & est. & $\mid t$-rat. $\mid$ \\
\hline$\alpha_{c_{1}}$ & 0.000 & fixed & 0.000 & fixed & 0.000 & fixed \\
\hline$\alpha_{c_{2-16}}$ & -0.140 & 23.61 & -0.084 & 23.48 & -0.058 & 14.44 \\
\hline$\alpha_{c_{17-31}}$ & - & - & -0.406 & 17.20 & -0.168 & 18.77 \\
\hline$\alpha_{c_{32-46}}$ & - & - & - & - & -0.760 & 11.19 \\
\hline$\beta_{\mathrm{AC}}$ & 0.299 & $11.8 \overline{4}$ & $0 . \overline{3} 12$ & 11.97 & $0 . \overline{3} \overline{1}$ & $\overline{1} \overline{2} . \overline{4}$ \\
\hline$\beta_{\mathrm{AS}}$ & 0.680 & 24.65 & 0.720 & 25.32 & 0.744 & 25.09 \\
\hline$\beta_{\mathrm{F}}$ & 0.299 & 11.85 & 0.306 & 11.76 & 0.317 & 12.09 \\
\hline$\beta_{\mathrm{G}}$ & 0.395 & 15.55 & 0.436 & 16.71 & 0.445 & 16.79 \\
\hline$\beta_{\mathrm{S}}$ & 0.555 & 20.39 & 0.588 & 21.27 & 0.611 & 21.31 \\
\hline$\beta_{\mathrm{SQ}}$ & -1.477 & 23.47 & -1.768 & 25.84 & -1.841 & 25.85 \\
\hline$\pi_{\alpha_{c_{1}}}$ & 0.594 & $\overline{8} . \overline{17}$ & $0 . \overline{3} 57$ & $2.4 \overline{1}$ & $0.09 \overline{7}$ & $0 . \overline{4} 4$ \\
\hline$\pi_{\alpha_{c_{2-16}}}$ & 0.406 & 5.58 & 0.478 & 2.97 & 0.618 & 2.36 \\
\hline$\pi_{\alpha_{c_{17-32}}}$ & - & - & 0.164 & 5.27 & 0.219 & 2.51 \\
\hline$\pi_{\alpha_{c_{32-46}}}$ & - & - & - & - & 0.066 & 2.99 \\
\hline$\pi_{\alpha_{l_{3}} \neq 0}$ & $0.07 \overline{3}$ & $\overline{1} . \overline{0} 2$ & $0 . \overline{2} \overline{6} 4$ & $\overline{1} .8 \overline{1}$ & $\overline{0} \overline{4} \overline{7} \overline{0}$ & $2 . \overline{17}$ \\
\hline$\pi_{\alpha_{l_{3,6}} \neq 0}$ & 0.037 & 0.97 & 0.021 & 1.08 & 0.047 & 1.11 \\
\hline$\pi_{\alpha_{l_{3,6,12}} \neq 0}$ & 0.005 & 0.40 & 0.010 & 1.11 & 0.005 & 0.43 \\
\hline$\pi_{\alpha_{l_{3,6,12,24}} \neq 0}$ & 0.001 & 0.25 & 0.008 & 1.30 & 0.007 & 0.92 \\
\hline$\pi_{\alpha_{l_{3,6,12,24,48}}} \neq 0$ & 0.124 & 4.30 & 0.131 & 4.06 & 0.124 & 2.72 \\
\hline$\pi_{\alpha_{l_{6}} \neq 0}$ & 0.001 & 0.25 & 0.002 & 0.21 & 0.003 & 0.21 \\
\hline$\pi_{\alpha_{l_{6,12}} \neq 0}$ & 0.001 & 0.25 & 0.000 & 0.23 & 0.001 & 0.22 \\
\hline$\pi_{\alpha_{l_{6,12,24}} \neq 0}$ & 0.001 & 0.25 & 0.001 & 0.21 & 0.001 & 0.19 \\
\hline$\pi_{\alpha_{l_{6,12,24,48}} \neq 0}$ & 0.018 & 0.65 & 0.008 & 0.76 & 0.014 & 0.59 \\
\hline$\pi_{\alpha_{l_{12}} \neq 0}$ & 0.001 & 0.25 & 0.001 & 0.25 & 0.001 & 0.24 \\
\hline$\pi_{\alpha_{l_{12,24}} \neq 0}$ & 0.007 & 1.08 & 0.011 & 0.82 & 0.009 & 0.81 \\
\hline$\pi_{\alpha_{12,24,48} \neq 0}$ & 0.062 & 2.83 & 0.121 & 3.32 & 0.123 & 2.37 \\
\hline$\pi_{\alpha_{l_{24} \neq 0}}$ & 0.002 & 0.28 & 0.001 & 0.27 & 0.001 & 0.21 \\
\hline$\pi_{\alpha_{l_{24,48}} \neq 0}$ & 0.023 & 1.69 & 0.045 & 1.77 & 0.076 & 2.20 \\
\hline$\pi_{\alpha_{l_{48}} \neq 0}$ & 0.053 & 4.32 & 0.020 & 1.74 & 0.023 & 1.58 \\
\hline $\mathcal{L}(\hat{\beta})$ & \multicolumn{2}{|c|}{$-7,315.88$} & \multicolumn{2}{|c|}{$-7,056.27$} & \multicolumn{2}{|c|}{$-6,976.31$} \\
\hline$K$ & \multicolumn{2}{|c|}{22} & \multicolumn{2}{|c|}{38} & \multicolumn{2}{|c|}{54} \\
\hline $\bar{\rho}^{2}$ & \multicolumn{2}{|c|}{0.331} & \multicolumn{2}{|c|}{0.353} & \multicolumn{2}{|c|}{0.359} \\
\hline AIC/N & \multicolumn{2}{|c|}{1.470} & \multicolumn{2}{|c|}{1.421} & \multicolumn{2}{|c|}{1.408} \\
\hline $\mathrm{BIC} / \mathrm{N}$ & \multicolumn{2}{|c|}{1.486} & \multicolumn{2}{|c|}{1.449} & \multicolumn{2}{|c|}{1.447} \\
\hline
\end{tabular}


14 additional parameters, providing a significant likelihood ratio test statistic.

While PDP(T)-1 allows for heterogeneous patterns of both cost thresholds and cut-offs, it assumes homogeneous cost sensitivities among respondents who considered any cost levels. Since this is a somewhat stringent assumption, in PDP(T)2 we obtain two values to describe the distribution in cost sensitivities among the subset of respondents where cost had a bearing on their choices. We achieve this in PDP(T)-2 by introducing a further 15 latent classes that are similar in nature to classes 2-16 in PDP(T)-1, but the estimated cost coefficient in classes 17-31 takes a different value from that obtained in classes 2-16. One of the most striking impacts of allowing for this heterogeneity is the further substantial drop in the probability associated with the class in which the cost attribute had no influence on the choices made by respondents. Under this specification it is implied that around 35 percent of respondents completely ignored cost. Instead, adding together the predicted probabilities for classes 2-16 reveals that almost half of respondents are estimated with a moderate marginal utility of money and that approximately 16 percent of respondents are identified as belonging to classes 17-32, which has a relatively high marginal utility of money. This model predicts that around 13 percent of respondents attended to all cost levels, meaning that just over half of respondent exhibited patterns of cost thresholds and cut-offs. The lowest cost level is predicted as having been considered by 43 percent, which includes a large subset of respondents (over one-quarter) who only considered this cost level. In contrast, the second lowest cost level was found to be considered by 18 percent and the respective figures for the three highest cost levels are all around 30 percent. We remark that 7 percent of respondents attended to only the highest two cost levels, but that this increases to over 20 percent when we calculate the proportion of respondents who only considered all but the lowest level. We note that of the 32 percent of respondents who attended to all but the highest cost level, most of it is due to the high proportion who attended to only the lowest cost level. We find that the likelihood ratio statistic of 127.22 against the $\chi^{2}$ critical value of $41.34\left(\chi_{28,0.05}^{2}\right)$, confirms that an improvement in model fit is achieved as one moves from PDP-2 to PDP(T)-2.

Our final model, PDP(T)-3, attempts to accommodate further heterogeneity in cost sensitivities by introducing an additional 15 classes. Results attained from this model reveal a further decline in the proportion of respondents completely disregarding cost as more heterogeneity is accounted for. Indeed, we find that less than 10 percent of respondents ignore all cost levels, which is in complete contrast to the predictions of around 70 percent under the LC and PDP model specifications. Over 60 percent of respondents are found as having relatively modest marginal utility from money. The two further subsets of respondents of approximately 22 and 7 percent are found as having moderately high and extremely high cost sensitivity, respectively. The proportion of respondents found to have considered all cost levels is in line with the previous two models. Remarkably, however, 
almost half of respondents focused solely on the lowest cost level. The model results also imply that the lowest cost level was considered by almost two-thirds of respondents, which is somewhat higher than the respective figures for the $€ 6$ level (20 percent), $€ 12$ level ( 28 percent) and $€ 24$ and $€ 48$ levels (36 percent for each). We note that this model is associated with the highest log-likelihood and that a comparison of the $\bar{\rho}^{2}$, AIC and BIC statistics confirm this finding generally stands even after accounting for the loss of parsimony.

Again, the findings under the PDP(T) models point towards possible confounding between heterogeneity in cost sensitivities and non-attendance. Accommodating for more of this heterogeneity, especially where heterogeneous patterns of cost thresholds and cut-offs are dealt with simultaneously, leads to a very substantive drop in the proportions of respondents predicted as having completely ignored cost - to the extent that the phenomenon almost disappears. These models also highlight that the often invoked assumption that respondents attend to all cost levels in the same manner is likely to be erroneous. The advantage of the PDP(T) models is that only the cost levels that have actually influenced the respondent's sequence of choices enter the log-likelihood function. Indeed, in our empirical dataset we find that the PDP(T) models lead to a better interpretation of the role that the different cost levels played on respondent's choices, which should, ultimately, be advantageous when it come to reaching welfare conclusions.

\subsection{Cost thresholds, cut-offs and sensitivities}

While Table 3 reports the aggregate probabilities for each pattern of cost threshold and cut-off, it is also of interest to explore if there are differences across the subsets of respondents with varying cost sensitivities. For this reason, in Figure 1 we present mosaic plots representing the predicted breakdown of the cost threshold and cut-off patterns adopted by respondents for each of the estimated cost coefficient under the three threshold models. The increasing column widths clearly illustrate that the proportion of the sample predicted with a non-zero cost coefficient increases as one moves from PDP(T)-1 to PDP(T)-3. Focusing on the plot associated with PDP(T)-2 reveals that the predicted patterns of cost thresholds and cut-offs are noticeably different between the larger subset who were relatively cost insensitive and the smaller subset who were more sensitive to it. We observe that the cost insensitive subset were more likely to focus solely on the lower cost levels. ${ }^{2}$ Indeed, over half of this subset are estimated as having focused solely on the

\footnotetext{
${ }^{2}$ We recognise that, although the estimated cost coefficient obtained for this subgroup would suggest that these respondents are relatively cost insensitive, an examination of the predicted patterns of cost thresholds and cut-offs highlights that many of these respondents disregarded the higher cost levels. It could, thus, be interpreted that these respondents were actually highly cost sensitive and the lowly estimated (in absolute terms) cost coefficient is due to the fact that the lower cost levels had a greater bearing on their choice than the higher cost levels. Indeed, this finding further demonstrates the merits of our PDP(T) models for gaining a better insight into the behavioural
} 


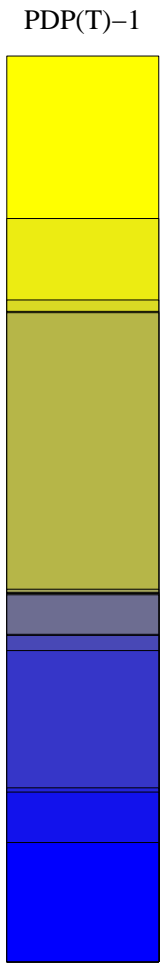

$\alpha_{c_{2-16}}$
$\operatorname{PDP}(\mathrm{T})-2$

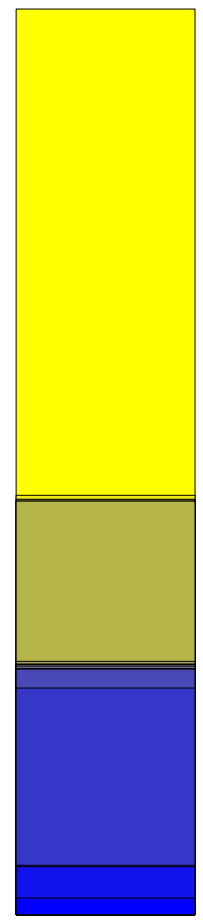

$\alpha_{c_{2-16}}$

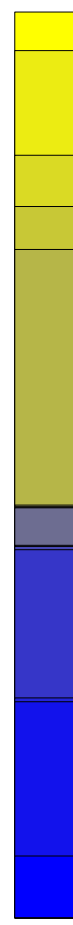

$\alpha_{c_{17-31}}$
$\operatorname{PDP}(\mathrm{T})-3$

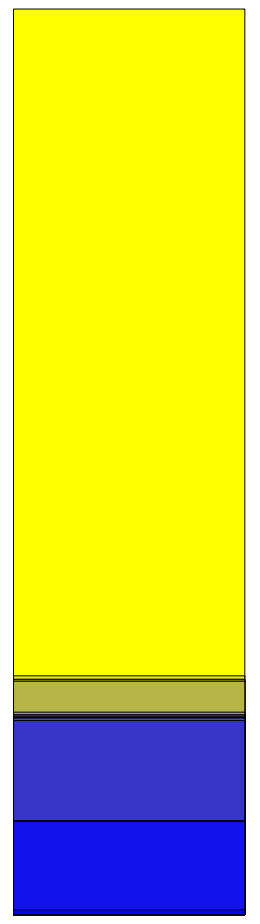

$\alpha_{c_{2-16}}$

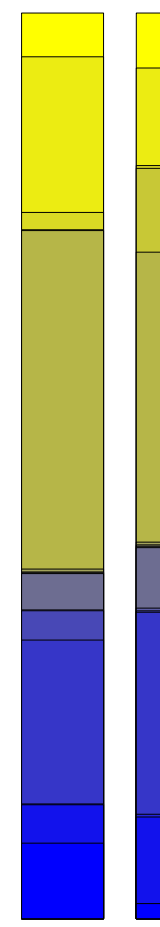

$\alpha_{c_{17-31}} \alpha_{c_{32-46}}$

$$
\begin{aligned}
& \square \quad \alpha_{l_{3}} \neq 0 \\
& \square \quad \alpha_{l_{3,6}} \neq 0 \\
& \square \quad \alpha_{l_{3,6,12}} \neq 0 \\
& \square \quad \alpha_{l_{3,6,12,24}} \neq 0 \\
& \square \quad \alpha_{l_{3,6,12,24,48}} \neq 0 \\
& \square \quad \alpha_{l_{6}} \neq 0 \\
& \square \quad \alpha_{l_{6,12}} \neq 0 \\
& \square \quad \alpha_{l_{6,12,24}} \neq 0 \\
& \square \quad \alpha_{l_{6,12,24,48}} \neq 0 \\
& \square \quad \alpha_{l_{12}} \neq 0 \\
& \square \quad \alpha_{l_{12,24}} \neq 0 \\
& \square \quad \alpha_{l_{12,24,48}} \neq 0 \\
& \square \quad \alpha_{l_{24}} \neq 0 \\
& \square \begin{array}{l}
\alpha_{l_{24,48}} \neq 0 \\
\alpha_{l_{48}} \neq 0
\end{array}
\end{aligned}
$$

Figure 1: Predicted cost threshold and cut-off patterns

lowest cost level (the respective figure for the cost sensitive subset is less than 5 percent). Inspection of the mosaic plot suggests that the cost sensitive subset had a greater propensity of considering all cost levels and gave considerably more attention to the higher cost levels. This is consistent with the fact that cost sensitive respondents are likely to consider all cost levels, especially higher ones. Turning our attention to the mosaic plot produced from the PDP(T)-3 membership probabilities we note how this reveals differences in the patterns of cost thresholds and cut-offs adopted by respondents identified with different cost coefficients. Similar to the inferences reached under PDP(T)-2, we note that the largest of the three subgroups, who are associated with the lowest cost sensitivity, appear to have been more inclined to ignore the higher cost levels. Overall, almost three-quarters of this subgroup considered only the lowest two cost levels, compared to 22 percent and 17 percent for the second and third subsets of respondents respectively who were also estimated with a non-zero cost coefficient. Interestingly, aside from slightly higher predictions of attendance to the higher cost levels, we observe that the patterns of cost thresholds and cut-offs exhibited by the two smaller subsets are quite similar in nature.

aspects of respondent's choices. 


\subsection{Welfare estimation results}

An alternative way of teasing out the effects of cost thresholds, cut-offs and sensitivities is to consider the effects on the MWTP estimates. Table 4 compares the annual MWTP results to conserve each of the fish species under the nine model specifications. We note that the reported MWTP estimates are weighted to account for the heterogeneous sensitivities to cost. For the PDP and PDP(T) models it is also necessary to account for the fact that it is not possible to derive a marginal WTP estimate for the subset identified as having zero marginal utility for the cost attribute. For this reason, we only compute MWTP estimates for classes with a non-zero cost coefficient. Therefore, the calculation for the unconditional (or marginal) MWTP associated with attribute $k$ is obtained as follows:

$$
\operatorname{MWTP}_{k}=\sum_{\forall \alpha_{c} \neq 0}\left(\frac{\pi_{c}}{1-\pi_{\alpha_{c}=0}} \times \frac{\beta_{k}}{-\alpha_{c}}\right) .
$$

A comparison of the MWTP estimates reveals that the implied ordering is somewhat consistent across the nine specifications (i.e., respondents were generally willing to pay most for preserving Altantic salmon and sonaghan, and least

Table 4: MWTP estimates ( $€$ s per year with standard errors in parentheses)

\begin{tabular}{|c|c|c|c|c|c|c|}
\hline & $\mathrm{AC}$ & AS & $\mathrm{F}$ & $\mathrm{G}$ & $\mathrm{S}$ & $\pi_{\alpha_{c} \neq 0}$ \\
\hline \multirow{5}{*}{$\begin{array}{c}\text { MNL } \\
\text { LC-2 } \\
- \\
\text { LC-3 }\end{array}$} & $\begin{array}{l}17.15 \\
(1.26)\end{array}$ & $\begin{array}{l}36.28 \\
(1.49)\end{array}$ & $\begin{array}{l}16.76 \\
(1.26)\end{array}$ & $\begin{array}{l}20.68 \\
(1.26)\end{array}$ & $\begin{array}{l}28.68 \\
(1.38)\end{array}$ & 1.000 \\
\hline & -139.19 & $-\overline{3} \overline{1} 5.08$ & $-\overline{1} \overline{4}-\overline{4}$ & -186.72 & $-\overline{258.94}$ & \\
\hline & (12.21) & (15.98) & (12.22) & (12.49) & (14.43) & 1.000 \\
\hline & $-21.3 \overline{3}$ & $-4 \overline{7} . \overline{35}$ & -21.14 & -28.36 & -39.34 & \\
\hline & $(2.35)$ & (4.04) & $(2.34)$ & $(2.75)$ & $(3.51)$ & 1.000 \\
\hline PDP-1 & 2.47 & 5.60 & 2.50 & 3.29 & 4.59 & 0.282 \\
\hline & $(0.2)$ & $(0.22)$ & $(0.2)$ & $(0.2)$ & $(0.22)$ & \\
\hline PDD & $2.77^{-}$ & 6.19 & 2.78 & 3.65 & 5.11 & \\
\hline PDP-2 & $(0.23)$ & $(0.28)$ & $(0.23)$ & $(0.23)$ & $(0.26)$ & \\
\hline & $-\overline{3 .}-$ & -7.29 & ${ }^{-} 3 . \overline{31}$ & $\overline{4} . \overline{26}$ & $6.04^{-}$ & \\
\hline PDP-3 & $(0.26)$ & $(0.37)$ & $(0.27)$ & $(0.28)$ & $(0.34)$ & 0.357 \\
\hline PDP(T)-1 & 2.13 & 4.48 & 2.13 & 2.81 & 3.95 & 0.406 \\
\hline & $(0.17)$ & $(0.2)$ & $(0.17)$ & $(0.17)$ & $(0.19)$ & \\
\hline $\mathrm{PDP}(\mathrm{T})-2$ & $1 . \overline{53}$ & 3.52 & $1.50^{-}$ & $2 . \overline{13}$ & $2.88^{-}$ & 0643 \\
\hline$\Gamma(1)-2$ & $(0.3)$ & $(0.63)$ & $(0.3)$ & $(0.39)$ & $(0.53)$ & 0.643 \\
\hline PDP(T)-3 & 3.96 & 8.92 & 3.80 & 5.34 & $7 . \overline{32}$ & 0003 \\
\hline PDP(1)-3 & $(0.88)$ & (1.88) & $(0.84)$ & (1.15) & (1.54) & 0.903 \\
\hline
\end{tabular}


for Arctic char and ferox). The most striking finding arising from the MWTP analysis is the fact that the MNL model produces values of a much higher magnitude (approximately ranging between $€ 17$ and $€ 36$ ), suggesting the influential role of ignoring heterogeneity in both cost preferences and processing strategies when it comes down to welfare measure estimation. However, considering the heterogeneity in cost sensitivities alone leads to highly negative MWTP estimates, which implies that, on average, the sample of respondents are actually willing to pay to ensure the fish are not conserved, which is not defensible. We do remark though, contrasting the findings under LC-2 and LC-3 suggests that the derived MWTP estimates become less extreme when more of the distribution in cost sensitivities is accounted for. The finding of negative MWTP does not comply with our expectations, but is an artefact of the positive, and lowly, estimated cost coefficient in the largest latent classes in both LC- 2 and LC-3 models, a fact that clearly suggests a mis-specification. While under both of these models the lowly estimated cost coefficients are interpreted as preference heterogeneity, it is almost certainly to be a consequence of non-attendance. This provides clear evidence for the need to distinguish preference heterogeneity and processing strategies to ensure appropriate interpretation. This is especially the case for the cost attribute, since its parameter appears in the denominator of the MWTP calculation, which, as we have shown here, can result in extreme and implausible estimates. Nevertheless, as can be seen in the final column in Table 4, accounting for the non-attendance associated with the cost attribute comes at the expense of only being able to retrieve MWTP estimates for a subset of the sample. Notwithstanding this limitation, doing so should raise the degree of confidence that the MWTP estimates are not sensitive to respondents ignoring cost and are based on the relevant subset of respondents who did attend to the cost attribute.

Under all models that address cost non-attendance, we observe that the MWTP estimates are relatively comparable (all estimates range between $€ 1.50$ and $€ 9$ ). We find that allowing for more heterogeneous cost sensitivities leads to a slight increase in the MWTP estimates. This is linked to the increased proportion of respondents from which these estimates are derived (i.e., models explaining more of the preference heterogeneity are better equipped to disentangle relatively cost insensitive respondents from those not attending to cost at all). The models that account for cost thresholds and cut-offs provide MWTP estimates that are in line with those that assume respondent's propensity to disregard the cost information is the same, irrespective of its level. The main difference is the fact that the MWTP estimates based on models taking patterns of cost thresholds and cut-offs are derived from a much larger share of the sample. This should generate more confidence in the implied welfare estimates by these models. 


\section{Conclusions}

Unlike previous attempts made to uncover the incidence of non-attendance among respondents, in this paper we do not assume that the processing strategy of nonattendance applies to the attribute independent of its levels. Instead, we facilitate the fact that respondents are likely to exhibit patterns of using thresholds and cut-offs, in which case they will disregard the attribute only when its level falls outside of the level consideration set defined by specific thresholds. We develop a probabilistic decision process model (similar in form to a latent class model, but where we define each class to describe a specific heuristic) to facilitate the complete range of cost thresholds that may be held by respondents. Moreover, in an attempt to address the potential confounding between taste heterogeneity and non-attendance, our model simultaneously allows for a segmentation of respondents based on their sensitivities to cost and their adoption of cost thresholds.

Results based on a stated choice experiment exploring the existence value of rare fish species in Ireland, provide further confirmation that a share of respondents do not attend to all attributes, and in particular excluded the cost attribute from their decision-making process. Importantly, however, when the range in cost thresholds, cut-offs and sensitivities are accounted for the incidence of complete non-attendance is found to be markedly lower, to the extent that it almost disappears (e.g., we observe a drop from over 70 percent under our naïve nonattendance model to less than 10 percent under our best fitting model). While specific to this dataset, this result highlights that the problem of respondents ignoring cost (or, indeed, any other attribute) may not be as widespread a problem as previously thought. While a share of the sample are still probabilistically identified as having ignored the cost at least at some levels, our approach nevertheless provides a framework for retrieving a cost coefficient from almost the entire sample, which is robust and not influenced by non-attendance.

Importantly, as shown in the paper, including variables within the model that played no role in respondent's decisions led to erroneous predictions and inappropriate policy conclusions. Our analysis provides evidence for the need to disentangle heterogeneity in cost sensitivities and heterogeneity in the adoption of processing strategies linked to threshold levels. Results further show that accounting for both types of heterogeneity leads to substantial gains in model fit and that failing to concurrently account for both has serious implications welfare analysis. Crucially, our analysis reveals that allowing for more flexible processing strategies and greater variations in cost sensitivities greatly facilitates the calculation of welfare estimates from a greater share of the sample.

Results in this paper highlight the importance of identifying the cost levels that actually influence choices. While our analysis shows this from the welfare estimation point of view, knowledge of the cost levels that actually have an influence on respondent's choices should also help at the experimental design stage. In- 
deed, implementing this type of analysis to data collected during piloting should ensure that the levels of cost used in the main survey are behaviourally relevant. Our findings also provide compelling evidence for further research in this area. Future studies should incorporate procedures for identifying and dealing with the heterogeneity in tastes as well as in processing so that the sensitivity on model performance and welfare estimates can be further evaluated. 


\section{References}

Campbell, D., Aravena, C. D. and Hutchinson, W. G. (2011). Cheap and expensive alternatives in stated choice experiments: are they equally considered by respondents?, Applied Economics Letters In press.

Campbell, D., Hensher, D. A. and Scarpa, R. (2011). Non-attendance to attributes in environmental choice analysis: a latent class specification, Journal of Environmental Planning and Management .

Campbell, D. and Hess, S. (2011). Outlying sensitivities in discrete choice data: consequences and remedies, Under review.

Campbell, D., Hess, S., Scarpa, R. and Rose, J. M. (2010). Accommodating coefficient outliers in discrete choice modelling: a comparison of discrete and continuous mixing approaches, in S. Hess and A. Daly (eds), Choice Modelling: The State-of-the-art and the State-of-practice - Proceedings from the Inaugural International Choice Modelling Conference, Emerald Group Publishing, Bingley, pp. 331-352.

Campbell, D., Hutchinson, W. G. and Scarpa, R. (2008). Incorporating discontinuous preferences into the analysis of discrete choice experiments, Environmental and Resource Economics 41: 401-417.

Cantillo, V., Heydecker, B. and Ortúzar, J. D. (2006). A discrete choice model incorporating thresholds for perception in attribute values, Transportation $R e$ search Part B 40: 807-825.

Cantillo, V. and Ortúzar, J. D. (2006). Implications of thresholds in discrete choice modelling, Transport Reviews 26: 667-691.

Carlsson, F., Kataria, M. and Lampi, E. (2010). Dealing with ignored attributes in choice experiments on valuation of Sweden's Environmental Quality Objectives, Environmental and Resource Economics 47: 65-89.

Chou, H. Y., Lu, J. L. and Fu, C. (2008). The study of price accept threshold for the "Blue Highway" tour of the North-East Region in Taiwan, Journal of Marine Science and Technology 16: 255-264.

DeShazo, J. R. and Fermo, G. (2002). Designing choice sets for stated preference methods: the effects of complexity on choice consistency, Journal of Environmental Economics and Management 2002: 123-143.

Han, S., Gupta, S. and Lehmann, D. R. (2001). Consumer price sensitivity and price thresholds, Journal of Retailing 77: 435-456.

Hensher, D. A. (2010). Attribute processing, heuristics, and preference construction in choice analysis, in S. Hess and A. Daly (eds), Choice Modelling: The State-of-the-art and the State-of-practice - Proceedings from the Inaugural International Choice Modelling Conference, Emerald Group Publishing, Bingley, pp. 35-70.

Hensher, D. A. and Greene, W. H. (2010). Non-attendance and dual processing of common-metric attributes in choice analysis: a latent class specification, Empirical Economics 39: 413-426. 
Hensher, D. A. and Rose, J. M. (2011). The influence of alternative acceptability, attribute thresholds and choice response certainty on automobile purchase preferences, Institute of Transport and Logistic Studies, University of Sydney .

Hensher, D. A., Rose, J. M. and Greene, W. H. (2005). The implications on willingness to pay of respondents ignoring specific attributes, Transportation 32: 203-222.

Hensher, D. A., Rose, J. M. and Greene, W. H. (2011). Accounting for endogeneity of attribute non-attendance in willingness to pay: a note and a warning for stated choice experiment design, Institute of Transport and Logistic Studies, University of Sydney .

Mørkbak, M. R., Christensen, T. and Gyrd-Hansen, D. (2010). Choke price bias in choice experiments, Environmental and Resource Economics 45: 537-551.

Puckett, S. M. and Hensher, D. A. (2008). The role of attribute processing strategies in estimating the preferences of road freight stakeholders, Transportation Research Part E 44: 379-395.

Sælensminde, K. (2006). Causes and consequences of lexicographic choices in stated choice studies, Ecological Economics 59: 331-340.

Scarpa, R., Gilbride, T. J., Campbell, D. and Hensher, D. (2009). Modelling attribute non-attendance in choice experiments for rural landscape valuation., European Review of Agricultural Economics 36: 151-174.

Scarpa, R. and Rose, J. M. (2008). Designs efficiency for nonmarket valuation with choice modelling: how to measure it, what to report and why, Australian Journal of Agricultural and Resource Economics 52: 253-282.

Scarpa, R., Thiene, M. and Hensher, D. A. (2010). Monitoring choice task attribute attendance in nonmarket valuation of multiple park management services: does it matter?, Land Economics 86: 817-839.

Swait, J. (2001). A non-compensatory choice model incorporating attribute cutoffs, Transportation Research Part B 35(10): 903-928.

Vermeulen, B., Goos, P., Scarpa, R. and Vandebroek, M. (2011). Bayesian conjoint choice designs for measuring willingness to pay, Environmental and Resource Economics 48: 129-149. 